DOI: https://doi.org/10.46296/gt.v5i9edespmar.0054

\title{
EL LUGAR DEL CUERPO Y SU IMPACTO EN LAS SESIONES DE PSICOTERAPIA CON MODALIDAD VIRTUAL: DESAFÍOS DE LA PSICOTERAPIA VIRTUAL CON ORIENTACIÓN PSICOANALÍTICA
}

\section{THE PLACE OF THE BODY AND ITS IMPACT ON PSYCHOTHERAPY SESSIONS WITH VIRTUAL MODALITY: CHALLENGES OF VIRTUAL PSYCHOTHERAPY WITH PSYCHOANALYTIC ORIENTATION}

\author{
Andrade-Castro José Abelardo ${ }^{\text {; }}$ : Egas-Reyes Verónica ${ }^{2}$ \\ ${ }^{1}$ Estudiante de la Maestría Académica con Trayectoria en Investigación en Psicología, Mención \\ Psicoterapia, Instituto de Posgrado, Universidad Técnica de Manabí. Portoviejo, Ecuador. \\ Correo: jandrade9559@utm.edu.ec. ORCID ID: https://orcid.org/0000-0002-2360-3926 \\ 2 Pontificia Universidad Católica del Ecuador. Portoviejo, Ecuador. Correo: \\ megas776@puce.edu.ec. ORCID ID: https://orcid.org/0000-0002-5361-8213
}

\begin{abstract}
Resumen
El presente artículo hace una revisión teórico - conceptual, sobre el lugar del cuerpo en las sesiones de psicoterapia virtual. Para este fin se hace una revisión bibliográfica de diversos textos, artículos y documentos con relación en primera instancia con los antecedentes históricos del cuerpo como objeto de estudio desde distintos campos de las ciencias psicológicas y el psicoanálisis; posteriormente se hace una reseña de los postulados más relevantes desde la teoría psicoanalítica de Jacques Lacan, señalando los tres registros del psiquismo: lo real, lo simbólico y lo imaginario, para desde esta perspectiva poder comprender el lugar que tiene el cuerpo más allá de un discurso biomédico. Por otra parte, se describe, cómo los fenómenos transferenciales se manifiestan en las sesiones de psicoterapia en el campo de lo virtual debido a la presencia virtual del cuerpo del psicoterapeuta. Para finalizar se reseña las diversas opiniones de psicólogos y psicoanalistas, con artículos de referencia sobre el impacto que genera en las sesiones de psicoterapia virtual, la ausencia de lo presencial del cuerpo, y cómo esta forma de vincularse, influye en la dinámica de la psicoterapia. Este artículo resalta cómo esta dinámica de la presencia - ausencia del cuerpo físico en las sesiones de psicoterapia virtual de orientación psicoanalítica puede llegar a impactar en el procedimiento psicoterapéutico.
\end{abstract}

Palabras clave: cuerpo, psicoanálisis, virtual, tecnología, psicoterapia.

\begin{abstract}
This article makes a theoretical-conceptual review on the place of the body in virtual psychotherapy sessions. For this purpose, a bibliographic review of various texts, articles and documents is made in relation in the first instance to the historical background of the body as an object of study from different fields of psychological sciences and psychoanalysis; Subsequently, a review of the most relevant postulates from Jacques Lacan's psychoanalytic theory is made, pointing out the three registers of the psyche: the real, the symbolic and the imaginary, so that from this perspective we can understand the place that the body has beyond a biomedical speech. On the other hand, it is described how transference phenomena are manifested in psychotherapy sessions in the virtual field due to the virtual presence of the psychotherapist's body. Finally, the various opinions of psychologists and psychoanalysts are reviewed, with reference articles on the impact generated in virtual psychotherapy sessions, the absence of the presence of the body, and how this way of bonding influences the dynamics of psychotherapy. This article highlights
\end{abstract}

Información del manuscrito:

Fecha de recepción: 06 de enero de 2022.

Fecha de aceptación: 24 de febrero de 2022.

Fecha de publicación: 04 de marzo de 2022. 
how this dynamic of the presence - absence of the physical body in psychoanalytically oriented virtual psychotherapy sessions can impact the psychotherapeutic procedure.

Keywords: body, psychoanalysis, virtual, technology, psychotherapy.

\section{INTRODUCCIÓN}

La Pandemia trae consigo una serie de desafíos en todos los ámbitos de la sociedad mundial actual, posibles recesiones económicas, presencia de emergencias sanitarias globales, restricciones en la movilidad humana y en la libertad de circulación, son algunas de las áreas que están comprometidas hoy por hoy. Bajo este precepto los prestadores de salud mental deben estar atentos y preparados a gestionar de manera eficiente y pertinente, métodos que permitan a los sujetos poder tramitar estas experiencias que pueden llegar a desbordar las capacidades psíquicas y por ende llevar como resultado a un corto $\mathrm{y} / \mathrm{o}$ mediano plazo a la Ilamada Segunda Pandemia ahora en temas de Salud mental, tal como lo resalta (La Gaceta médica, 2020): “...Aunque se espera un aumento en los síntomas de ansiedad durante estas circunstancias extraordinarias, existe el riesgo de que prevalezca un número clínicamente relevante de personas con ansiedad, depresión y que participen en comportamientos perjudiciales (como suicidio y autolesiones) que con toda probabilidad incrementarán". Sin embargo, explican estos autores, que cabe destacar que el aumento del suicidio no es inevitable, especialmente con los esfuerzos nacionales de mitigación. Es probable que las consecuencias potenciales de una recesión económica en la salud mental sean profundas para las personas directamente afectadas y sus cuidadores. Los supervivientes corren el riesgo de sufrir un trastorno de estrés postraumático y depresión. Bajo esta premisa es necesario tener en consideración de manera urgente, investigaciones que aporten a crear todos los recursos necesarios para la atención en salud mental en todos sus niveles de intervención y con los recursos más óptimos.

Los profesionales preparados en el campo de la Psicoterapia cuentan 
con otro reto aparte de lo ya descrito anteriormente, este consiste en la aplicación de los medios virtuales de interacción humana, el uso de la tecnología como mediador de la relación entre sujetos, el cual coadyuva al ejercicio efectivo de la aplicación de las medidas sanitarias de bioseguridad y además como es de saber, este medio es ampliamente usado en la actualidad, como bien resalta (Vaimberg, 2005) “...debido a la gran difusión e incorporación de estos sistemas de comunicación especialmente en los niños, adolescentes y jóvenes (en el mes de marzo del año 2011 había 2.000 millones de usuarios de Internet en el planeta). Esta gran difusión en la población justifica el desarrollo de métodos psicoterapéuticos mediados tecnológicamente, permitiendo aprovechar los usos de las TIC e incidiendo sobre los sentidos de estos".

Por lo tanto es necesario y pertinente que los profesionales en el campo de la Salud Mental: psicólogos clínicos, psiquiatras $u$ otros profesionales, cuenten con los criterios $y$ herramientas necesarias para la aplicación de una psicoterapia virtual e ir diseñando un modelo de intervención en este campo y que responda a las necesidades del contexto.

\section{Trabajos relacionados}

El marco teórico para la revisión del presente artículo, tomamos en consideración los conceptos de la psicoterapia con orientación psicoanalítica. Método con el cual se brinda a los sujetos una escucha diferente, en donde es reconocido en su singularidad, el paciente es llamado por su nombre, escuchando su padecimiento en tanto su singularidad y de esta manera se intenta rescatar y hacer devenir una subjetividad, método que aparece contrario a los distintos modelos de psicoterapia que tienen como objetivo, la estandarización de un método para enfrentar el sufrimiento humano, dejando de un lado lo único e irrepetible de la vida subjetiva, tal como lo manifiesta (Bacile et al., 2015) : "El psicoanálisis, como sabemos, es contrario a este discurso, ya que, en su quehacer el psicoanalista, intenta producir una ruptura, un corte en relación con la uniformidad, se dirige, a través de la 
particularidad del síntoma, a la singularidad del goce. No hay por lo tanto un "para todos" en la felicidad". Es relevante realizar esta diferencia pues a partir de este precepto, vamos tejiendo las raíces epistemológicas de nuestro estudio, donde el enfoque de sujeto prioriza la escucha del caso por caso, más allá de técnicas y métodos que tiendan a uniformizar.

Por otra parte, la psicoterapia en cada uno de sus modelos teóricos de referencia debe intentar adecuar sus técnicas y procedimientos a las nuevas condiciones tales como el distanciamiento físico y el uso prioritario del teletrabajo, es más, en la actualidad se hace más que necesario hacer revisiones teóricas y metodológicas de los cambios en la estructura y los procesos del método psicoterapéutico. Bajo este precepto, la dinámica de la presencia-ausencia del cuerpo del psicoterapeuta, es de suma relevancia como resalta el análisis del mismo Bacile: “...distinguiendo y subrayando la importancia capital que le otorgamos a la presencia de los cuerpos, tanto del analista como del analizante; escenario donde se despliega la singularidad de un sujeto, donde la expectativa gira en torno a analizar las formaciones del inconsciente (actos fallidos, sueños, equívocos)" (Op Cit., p 3). Estas consideraciones aparentemente se tornan difíciles de pensar al hacer el intento de llevar este encuadre a la realidad virtual sabiendo que la regla fundamental de la psicoterapia ha sido la clásica relación vis a vis.

El autor de este articulo avizora dificultades futuras en el ejercicio de la práctica del psicoanálisis propiamente dicho, como lo señala de manera más explícita (Ons, et al., 2020) con la siguiente cita : "Creemos importante remarcar, que si bien no consideramos posible la práctica del psicoanálisis bajo una modalidad on-line, por las diversas razones que hemos intentado esclarecer en el trabajo, nucleadas principalmente en los dispositivos de los que se vale el psicoanálisis, insalvables en la dirección de la cura, que se generan con la presencia y el discurso del analista, cabría preguntarse qué uso es posible hacer de la red o que merecería ser rescatado". Mas sin embargo, luego el mismo autor resalta la posibilidad de que la realidad virtual puede ser de suma ayuda en la aplicación de 
otros dispositivos psicológicos tales como la psicoterapia, donde resalta la idea "...que al "consultorio virtual" lo equiparamos con un modo de "ayuda" entre otros". $\mathrm{Y}$, es en esta modalidad de ayuda entre otros donde entran los modelos de intervención con psicoterapia virtual.

En otro momento de la entrevista (Op Cit, 2020): “...Sabemos de la importancia de todo aquello que posibilite aliviar el sufrimiento, $y$ creemos que Internet, utilizada con cautela y responsabilidad, puede ser uno de estos medios".

Con esta premisa, el autor considera: "...todo aquello que encaje en la sintomatología, del "sujeto que consulta", de aquel que se sirve de lo que se ofrece es terapéutico. Todo puede tener un efecto terapéutico, cualquier acto, cualquier palabra que calme un síntoma. $Y$ puede cumplirse este objetivo mediante la mediación de la realidad virtual.

Por lo tanto, es la función del psicoterapeuta encausar el malestar del síntoma, el síntoma mismo, para que no cause dolor. En fin, escuchar aquello que habla. Escuchar al síntoma detrás de una cámara de video conferencia que sirva de mediador, a pesar de la distancia física entre el psicoterapeuta y el paciente.

Muy a pesar de la ausencia del cuerpo físico, en una sesión de psicoterapia con modalidad on line, vemos que existe la posibilidad de instaurar un procedimiento, si bien no desde el psicoanálisis propiamente dicho sino de la aplicación del método de psicoterapia con orientación psicoanalítica.

\section{METODOLOGÍA}

El artículo es un trabajo de revisión bibliográfica. Esta ha sido definida como "la operación documental de recuperar un conjunto de documentos 0 referencias bibliográficas que se publican en el mundo sobre un tema, un autor, una publicación o un trabajo específico. Es una actividad de carácter retrospectivo que nos aporta información acotada a un periodo determinado de tiempo" (Gálvez, 2001). Para este fin se realizó una búsqueda y recopilación de archivos desde fuentes secundarias (repositorios, artículos y entrevistas) 
relacionados al tema del Cuerpo desde el psicoanálisis.

Luego en la estrategia de búsqueda se seleccionaron las palabras claves (cuerpo, psicoanálisis, psicoterapia, virtualidad, tecnología), se organizó la recopilación de datos en un período comprendido entre los años 2015-2020, sin embargo vale recalcar que la psicoterapia virtual, es un método actual y novedoso, muy a pesar que se tenga referencia que hay décadas atrás donde ya se ejercía dicha práctica. Bajo esta premisa se incluyeron los textos bajo dos criterios: primero que tengan relación con el trabajo de la psicoterapia con orientación psicoanalítica y segundo que sean artículos de revistas indexadas 0 entrevistas a expertos en la materia. Se excluyeron artículos de psicoterapia con otras orientaciones metodológicas y también documentos descritos en blogs personales o de dudosa rigurosidad científica.

\section{DESARROLLO}

La virtualidad nos plantea varias interrogantes en los actuales momentos a los y las psicoterapeutas, dado a la masificación de las intervenciones bajo el método de psicoterapia virtual, nos vemos obligados a generar varios análisis en este sentido, temas tales como: el cuerpo como concepto y objeto de estudio en la historia, el cuerpo físico y el cuerpo virtual, los fenómenos de transferencia y contratransferencia en la virtualidad, la presencia ausencia del psicoterapeuta bajo modalidad virtual. Por esta razón vamos a iniciar con un recorrido histórico sobre el tema del cuerpo.

\section{Aproximaciones teóricas al concepto de Cuerpo: Una} Revisión Histórica

Vamos a hacer una revisión de los principales postulados que explican la concepción de cuerpo desde las ciencias médicas, la psicología y el psicoanálisis, pues vale resaltar que existen diferencias tanto conceptuales como epistémicas a lo largo del desarrollo tanto de las humanidades, la psicología científica y la medicina. Como resalta (Carballo \& Crespo, 2003): "En la filosofía griega se pueden encontrar las primeras referencias directas al cuerpo; pero de la mano de una perspectiva dualista se instala una concepción de lo humano escindida. 
Ya desde los griegos se le otorga al cuerpo el carácter de instrumento en manos de la razón, ocupando de este modo el lugar de la no razón. El cuerpo es ante todo materia, distinta y opuesta a la no materia entendida como razón, amor, inteligencia, espíritu, alma etc". Esta dualidad donde el sujeto es divisible es un paradigma que si bien en algunos campos ha sido superado, aún pervive en algunos espacios de la cultura colectiva.

Bajo esta premisa de la divisibilidad del cuerpo y la mente, una tenía un valor diferencial sobre la otra sustancia, como lo decía el mismo autor: "Desde Parménides se le concede a la no materia un status superior, el status del ser. La materia, en cambio, resulta un obstáculo para la trascendencia del ser; obstáculo que, sin embargo, en gran parte de la cosmovisión griega, es capaz de ser domesticado a tal punto de "colaborar" con la razón. Este es el papel que, en parte, se le asigna a la gimnástica 0 arte del gimnastés" (Op Cit, p. 18).

Otro momento histórico importante para hacer una revisión histórica del Cuerpo, lo que encontramos en la era moderna, en la cumbre del pensamiento dualista parece alcanzarse en la filosofía de los siglos XVII y XVIII. El pensamiento Cartesiano en Francia y por otro lado el idealismo dialéctico de Hegel en Alemania configuran un nuevo paradigma de la experiencia corporal. Como lo resalta el mismo autor: "Negando el aporte que al conocimiento puedan hacer los sentidos y afirmando la preeminencia de las ideas -producto de la razón- respecto de la realidad material, estas posturas contribuyen aún más, aunque no precisamente desde una óptica religiosa, a subordinar al cuerpo al espíritu, en suma, a entender al cuerpo como mero organismo, como la porción animal del humano" (Op Cit, p. 21). Este es otro paradigma que también, aún se mantiene en la consciencia colectiva, muy a pesar de que se invita a modificar paradigmas que invitan al pensamiento inter $y$ trandisciplinario. Notamos acá, como con el paso de los siglos la idea de un cuerpo concebido como Materia, reducido a un organismo, donde las experiencias corporales no son tomadas en cuenta desde un punto de vista integral y dialéctico, y sobre 
todo un cuerpo desprovisto de toda comprensión desde lo simbólico se mantiene.

En otro orden, son las Ciencias Médicas las que se apropian de este como objeto de estudio e investigación, y es entendido como un organismo biológico y su intervención se centra en él. Como resalta (Op Cit, p. 25) "Ontogenéticamente es un organismo que se desarrolla madurativamente teniendo en cuenta las leyes neurofisiológicas. Su método de investigación es por un lado el cadáver y por el otro el estudio del hombre vivo con la ayuda de la inspección exterior, es decir a la exploración de los enfermos. A través de esta exploración la medicina realiza una lectura clínica de fenómenos, pues atiende al conjunto de signos para buscar en ellos su diagnóstico y tratamiento". Es entonces una materia concreta del cual se van generando métodos para la comprensión de los diversos principios y leyes que rigen su funcionamiento sano y enfermo, y es en este sentido que se va $a$ ir desarrollando las ciencias médicas.

Más adelante la Psicología con un enfoque naturalista, también tiene que opinar sobre el cuerpo, tiene en cuenta las estructuras biológicas y se basa en ellas puesto que establece que la construcción del "esquema corporal" se elabora progresivamente al compás del desarrollo y la maduración nerviosa, pero no se queda con una explicación puramente anatómica, sino que plantea que además existe otra dimensión que la resume en el "esquema corporal" como una representación mental la cual no solo equivale a sentir nuestro cuerpo como piel, músculos, huesos, cartílagos; y que no es solo la imagen de las partes del cuerpo como un dibujo, sino que resume las experiencias corporales cognitiva, subjetiva y afectiva. Aquí notamos que a pesar que se da espacio y un lugar al fenómeno psíquico, aún se mantiene el enfoque de base donde el cuerpo, el cual es una materia que produce como efecto una nueva sustancia.

Sin embargo, mucho después tenemos como primer antecedente, un análisis del cuerpo más allá del reduccionismo biologicista, y lo vamos a encontrar en la filosofía Marxista, más específicamente desde el Materialismo histórico, 
donde el Cuerpo es "lugar donde se produce la explotación y la alienación"... "El cuerpo del obrero, reducido a máquina, a instrumento, es reificado de modo coherente con los fines de la producción capitalista industrial" (Op Cit, p. 30). Ya notamos acá donde el cuerpo tiene otra dimensión y es llevado como "objeto" de estudio al campo de las ciencias sociales.

Es más tarde con el Psicoanálisis, donde se va a retomar varios conceptos de la dialéctica, de la sociología y la antropología del Cuerpo para llevarlo a una dimensión conceptual, desde lo Simbólico como vamos a revisar en el siguiente apartado.

\section{El cuerpo desde el Psicoanálisis}

Para el psicoanálisis y sobre todo desde la lectura de Lacan, el cuerpo desde el registro de Lo Real equivale al organismo, a diferencia del Cuerpo (Unzueta, 2013): "el cual se construye como cuerpo a partir de la relación con el Otro del significante, que en un inicio por excelencia lo encarna la madre, relación que se establece no por la satisfacción de las necesidades vitales sino por la relación con el significante: horarios, sabores, ritmos, golpes, caricias, voces, miradas, olores, etc. Es en esta influencia del significante sobre el organismo, el cuerpo biológico deviene un cuerpo erógeno, es decir un cuerpo simbólico que se prestará como superficie topológica de inscripción a recibir la marca significante y hará síntoma". Es decir aquella materia orgánica toma un nombre, los significantes revisten por medio del deseo del Otro, hará emerger un sujeto constituido por el lenguaje.

Acá podemos ya tener una nueva postura pues, para el psicoanálisis el cuerpo es construido, pero es diferente de la construcción del esquema corporal de la psicología. $\mathrm{Si}$ bien el esquema corporal de la psicología es una construcción, lo hace con referencia a la maduración orgánica y sigue teniendo como base el organismo biológico. En cambio en el psicoanálisis se trata del cuerpo como construcción, pero no tiene nada que ver con un desarrollo madurativo ni evolutivo, sino tiene que ver con una construcción a partir de la incidencia del significante, en la relación con el Otro. 
Más adelante el mismo autor (Op Cit, p. 20) hace referencia de lo dicho Lacan sobre los tres registros de lo psíquico y donde se lo enlaza con el concepto de cuerpo:

"Dentro del psicoanálisis se definirá al cuerpo desde los tres registros de la experiencia psicoanalítica: lo real, lo simbólico y lo imaginario": Desde el registro de lo Real el cuerpo puede equipararse al organismo -carne, mucosas, entrañas, cavidades, fluidos- de la medicina. Cuando un ser viviente viene al mundo es un organismo, pero no un cuerpo, el cuerpo se construye en la relación con el Otro del significante: Antes de nacer este organismo se lo espera con un nombre, un sexo, esperanzas, sueños, ideales, es decir ya circula en un discurso. En consecuencia pierde esta condición de real y pasa a constituirse como sujeto. Al mismo tiempo la madre que encarna al Otro primordial irá erogeneizando este organismo a partir de determinados significantes horarios, sabores, caricias, miradas, olores, voces, golpes, etc.- que irán marcando el cuerpo del sujeto. Aquí vemos como se inserta este cuerpo de lo Real a la cultura, al universo de lo simbólico.
Desde el registro de lo Simbólico el cuerpo es como un investimento, primer objeto que se catectiza. Lo que viste son deseos, necesidades, exigencias, apetencias, placeres, goces. Es un cuerpo vacío, sin contenido, hecho sin órganos, cuerpo que se prestará como superficie de inscripción a recibir la marca significante y en el que se irán privilegiando ciertas zonas erógenas y circuitos pulsionales.

Desde el registro de lo Imaginario el cuerpo es la vivencia de una imagen unitaria, que brinda unidad al organismo fragmentado con el que el sujeto nace. El organismo fragmentado encuentra su unidad en la imagen; la cual en su papel estructurante organiza el cuerpo ubicándolo como cuerpo humano: como forma total, superficie, recinto, límite, contorno, que va a ser habitado, investido, vestido, recubierto por la libido. Así el cuerpo se constituye como recubrimiento libidinal trazando una organización erógena”.

Si hacemos un análisis preliminar sobre estos aportes del psicoanálisis Lacaniano, podemos manifestar dos cuestiones importantes: primero que lo que está en juego en las sesiones 
de Psicoterapia virtual es la ausencia del Cuerpo de la realidad, más no el Cuerpo desde la concepción simbólica e imaginaria, y por otra parte, se establece como el Cuerpo es una construcción de significantes que parte de la relación con otro, esta ausencia de cuerpo presente, tendrá nuevas vías de significación y resignificación, pues el espacio de psicoterapia (presencial o virtual), es un encuentro humanizante, donde en la relación transferencial se construyen nuevos significantes que vienen a recaer directamente sobre el Cuerpo, encontrando en dicho elemento nuevas vías de subjetivación.

Pero para poder señalar los impactos que se puedan generar sobre las sesiones de psicoterapia de modalidad virtual, a partir de este momento y como hemos visto en esta reseña, hablaremos de una "Ausencia de un Cuerpo de la Realidad", es decir sobre la Presencia real del psicoterapeuta (el Psicoterapeuta en físico), pero también nos deja otro lectura muy interesante, como el paciente genera una resignificación de dicha ausencia y como esto podría impactar en las sesiones de psicoterapia con modalidad virtual, por lo tanto y a continuación vamos a hacer una revisión sobre los conceptos de lo que Lacan desarrollo sobre la Presencia del Analista.

\section{De la presencia del psicoterapeuta} (EI cuerpo en la sesión de psicoterapia)

En esta revisión teórica y para comprender la importancia de la ausencia del cuerpo en las sesiones de psicoterapia, se hace un análisis del concepto denominado por Lacan "La presencia del analista" (Lacan, 1963). El cual: “...se refiere justamente a la cubierta imaginaria de un pedazo de real; este desencadena un movimiento pulsional", es decir la sola presencia física del psicoterapeuta viene a dar como resultante un valor simbólico para el paciente.

Para seguir en este análisis, notamos que la presencia del cuerpo está dada en la voz y en su mensaje que va contenido en la palabra, para citar: “... la palabra es ya una presencia hecha de ausencia, y de esta pareja modulada de presencia y ausencia nace el universo de 
sentido": (Lacan Op. Cit. p. 95). Aquí vemos que hace sentido la presencia de una palabra en sesión, en este caso una palabra mediatizada por una imagen en la pantalla, con distintos puntos de referencia en la mirada sin contacto directo, en una voz modulada por el decodificador de señales, en una imagen del rostro aumentada por el juego de luces y filtros.

Para esto en el estricto caso del psicoanálisis clásico y con el intento de hacer un paralelismo de este método con la psicoterapia, usando la figura del psicoterapeuta, diría (Green, 2003) citando a Freud en un hecho casi anecdótico como teórico:

“...La presencia del analista, esa presencia que da cuenta de que uno está en el diván y se escucha la respiración del mismo. ¿Y Freud qué hacía? Era interesante la intervención de Freud, porque le decía: "usted está pensando en mí" y ya eso era volver a meterlo en el campo del significante... ¡Ya está, ya se olvidó de la respiración del analista!, la intervención de Freud es llevarlo al campo del semblante y está muy bien porque eso hace marchar el análisis, pero hay un punto en análisis que no se resuelve por la vía del semblante, sino que se resuelve por esta vía de lo real, diría del cuerpo del analista, en presencia y que luego vuelve a la pregunta del deseo del analista".

Acá podemos notar más claramente la idea argumentada anteriormente, como se da ese tránsito de "la cubierta imaginaria con un pedazo de lo real" al universo de lo simbólico, y si esta regla se cumple en el proceso de psicoterapia y, si la transferencia está bien instituida, sigue habiendo estrictamente dos cuerpos. Por lo tanto, este análisis es posible en las sesiones de psicoterapia, justamente porque en la modalidad virtual se puede tener acceso a la posición de escucha, la interpretación y la presencia del cuerpo del psicoterapeuta vía el objeto voz, lo cual hace posible un encuentro humanizante.

Pero es en esta voz donde se vehiculiza la palabra, una palabra que dice, y es el fundamento de la psicoterapia, además es en este decir, donde se va construyendo el vínculo terapéutico, pues "Toda palabra llama una respuesta" diría (Lacan,1975)". $Y$ es en este acto donde el encuentro paciente / psicoterapeuta ingresan a la relación 
de transferencia, transferencia necesaria para la dinámica de la psicoterapia con orientación psicoanalítica.

Pero no es suficiente la presencia en voz y la palabra, esta debe tener en retroalimentación una interrogante para completar el acto de la psicoterapia en ese escucha donde se emite una respuesta, tal como refiere (Novoa, 1999) "...Concomitante a la conformación de la interrogación subjetiva particular queda constituido el lugar de la escucha, de ese tercero que encarna la paradójica presencia de un sitio surgido desde el lugar de una ausencia. Porque es atravesando la pantalla de la imagen propia que el analizante al escuchar su relato enfrenta una nueva comprensión de lo vivido".

Como bien lo dice (Aryan, 2020) "...la relación virtual durante el uso de Skype, y debe ser cuidado con especial atención. La idea es que para pacientes con más debilidad y alteraciones yoicas, no siempre son necesarios reaseguramientos narcisistas. La presencia y constancia física del analista en el consultorio favorece ese efecto, pero mediante un dispositivo a distancia es necesario reforzar la presencia virtual armando una red confiable; por ejemplo, con el intercambio de mensajes de texto 0 de correo electrónico que contengan al yo para el momento en el que reciba la noticia de un señalamiento o la sorpresa de una interpretación, en una situación analítica restablecida.

Según las conceptualizaciones actuales, el psicoanálisis clínico siempre estuvo estructurado como un encuentro interpersonal en la consulta del psicoterapeuta y paciente o un grupo, como le conocemos en terapia de grupo 0 familiar. Sin embargo y referido al modelo de psicoterapia con orientación psicoanalítica. Se supondría que la distancia supone una limitación al método psicoanalítico, mas no a la aplicación de procedimientos de una psicoterapia psicoanalítica.

\section{DISCUSIÓN}

Debido a la pandemia nos hemos visto obligados no solo a intervenir mediante la lógica de lo virtual sino también a repensar el mismo método de la psicoterapia desde las 
condiciones que genera la realidad actual. La vigencia de los medios virtuales en el campo laboral, académico, para crear lazos sociales da cabida también a que se refuerce la aceptación de la psicoterapia con modalidad virtual.

Por otra parte, este tipo de psicoterapia incluso sirve para cumplir con las medidas preventivas de distanciamiento físico para prevenir el contagio de COVID.

En esta discusión partiendo desde lo simple a lo complejo, pasamos a un momento de análisis donde el Cuerpo más allá de lo biológico (lo concreto, presencial en la realidad del cuerpo del psicoterapeuta), por incidencia de los significantes en la relación con el otro (relación psicoterapeuta - paciente en la transferencia) y del Otro (el lugar donde deviene el universo de lo simbólico), este Cuerpo se anuda en los tres registros que propone Lacan (Cuerpo Real, simbólico e imaginario), estos conceptos metapsicológicos que parten si bien desde el psicoanálisis son de mucha utilidad para la psicología y la psicoterapia, pues permite tener un comprensión diferente, pues como sabemos este cuerpo se construye en esa relación mediante la palabra, los gestos, la voz, podríamos definirlo en primera instancia y como una primera interrogante que plantea esta revisión hablar de un Cuerpo virtual.

Si bien existe una ausencia de un cuerpo físico o más bien existe la presencia de un cuerpo virtual, el cuerpo para el psicoanálisis está presente en la palabra, en los gestos, en el tono de voz, verbigracia y en palabras de (Lévy, 1999) "introduce la noción de hipercuerpo para dar cuenta que en la virtualización el cuerpo se multiplica, "sale de sí mismo, adquiere nuevas velocidades, conquista nuevos espacios. Se vuelca al exterior y transforma la exterioridad técnica o la alteridad biológica en subjetividad concreta". Subjetividad concreta dada en estos fenómenos que hemos resaltado, como la entonación de una palabra o los movimientos en la cámara, la sonrisa o los gestos de aprobación/desaprobación van configurando la relación transferencial. Por tanto y siguiendo al mismo autor " el cuerpo virtualizado mantiene su entidad existencial, solo muta su identidad 
adoptando nuevas presencias, otras modalidades de ser y de operar en la multiplicidad de redes tecnológicas sin un lugar de referencia estable. Afirma que "la virtualización del cuerpo no es una desencarnación sino una reinvención, una reencarnación, una multiplicación".

Por otra parte, los fenómenos transferenciales tienen su lugar en la relación virtual, desde un modo particular, y en este sentido el paciente genera una resignificación de la ausencia del cuerpo físico del psicoterapeuta y como esta resignificación, podría impactar en las sesiones de psicoterapia con modalidad virtual tiene que ver más bien con la propia singularidad del paciente pero no entorpece que se dé la transferencia en psicoterapia, pues esta dinámica propia de la psicoterapia tiene sus elementos tradicionales bien establecidos tanto en la psicoterapia con modalidad presencial como en la virtual como lo es el encuadre.

Existe un fenómeno interesante que permite nuevos caminos de interrogación el cual esta demarcado como aquel lugar donde "se da el encuentro", y que en primera instancia esta en doble vía, el lugar (espacio físico) donde atiende el psicoterapeuta y el lugar donde recibe 'la intervención el paciente, y en esa lógica un tercer lugar o espacio transicional que en términos de Winnicot seria aquel espacio intermedio que no es ni el espacio exterior objetivo, ni el espacio interno subjetivo. Este tercer espacio se sitúa en la intersección de ambas realidades.

Esta zona intermedia de experiencia, no discutida respecto de su pertenencia a una realidad interna $o$ exterior (compartida), diría (Winnicot, 1992) que: "constituye la mayor parte de la experiencia del bebé, y se conserva a lo largo de la vida en las intensas experiencias que corresponden a las artes y la religión, a la vida imaginativa y a la labor científica creadora" y en su defecto en el proceso de psicoterapia.

En otros sentido y que resulta novedoso es que hay temas que en psicoterapia, las llamadas Psicoterapia de urgencia, donde puede existir riesgo vital y que desde la perspectiva psicodinámica, se sugiere: "La idea es que para 
pacientes con más debilidad y alteraciones yoicas, es necesario reforzar la presencia virtual armando una red confiable; por ejemplo, con el intercambio de mensajes de texto - de correo electrónico que contengan al yo para el momento en el que reciba la noticia de un señalamiento o la sorpresa de una interpretación, en una situación analítica restablecida. (Aryan, 2021). Esta idea es clave para que sirva de soporte a las intervenciones verbales dadas en lo virtual, acompañando con actividades de corte directivo. En otras palabras existen otras vías de comunicación en psicoterapia más allá de la videoconferencia que pueden acompañar a los dispositivos psicoterapéuticos virtuales y darle un importante apoyo.

Para finalizar existen fenómenos que están enmarcados en la lógica de lo no-todo, los limites, las imposibilidades, va marcando fallas, dificultades, tales como interrupciones en el audio, en la intermitencias de la señal, la caída de la Red, bloqueos, ralentización de la emisión y recepción de los mensajes, fenómenos que generan mayores cuestionamientos y brindan un prolífico campo de estudio a esta novedosa modalidad de psicoterapia donde esta mediatizada virtualmente.

\section{CONCLUSIONES}

Finalmente podemos concluir que el cuerpo va más allá de la concepción reduccionista, visto desde la materialidad, tal como ha sido descrito por la medicina, la filosofía y la misma psicología. Desde el psicoanálisis el cuerpo tiene un lugar en el campo del lenguaje y esto permite tener tanto un análisis diferente tanto a nivel teórico como en lo práctico pues permite al psicoterapeuta comprender la relación del cuerpo en la virtualidad.

La escucha diferente, la voz, la interpretación, la mirada son elementos que vienen a humanizar la relación psicoterapeuta - paciente y se construye este cuerpo tal como lo describió Lacan en los tres registros (Real, Simbólico e imaginario), dando paso con esto a la formulación de los fenómenos de transferencia y contratransferencia, vital para la consecución de todo procedimiento de psicoterapia.

La psicoterapia virtual no viene a tomar el lugar de la psicoterapia 
presencial sino más bien viene a ocupar un lugar que la sociedad demanda, motivo del contexto sanitario actual y de la masificación del uso de los medios tecnológicos, este dispositivo psicológico tiene sus propias particularidades, sin embargo, resulta de utilidad debido a como hemos revisado, cuenta con los criterios para que se pueda establecer la dinámica que facilita la relación psicoterapeuta - paciente, llevar la estructura de la sesión, y la presencia de un cuerpo en este caso un cuerpo virtual.

\section{BIBLIOGRAFÍA}

Antonietti, A (2018). Cuerpo VirtualCuerpo Real, en la Virtualización de las Organizaciones. Anuario de Investigaciones de la Facultad de Psicología, Instituto de Administración, Facultad de Ciencias Económicas

Aryan, A. (2020). Reconsideración del encuadre a la luz de las modificaciones tecnológicas. Reconsideración de la transferencia-

contratransferencia. Trabajo presentado en el 46th Congress 2009:

Psychoanalytic Practice: Convergence and Divergence de la Asociación
Psicoanalítica Internacional, Chicago.

Baringoltz, S. (2005). La importancia del estilo personal del terapeuta y el vínculo como herramientas terapéuticas. International Congress of Cognitive Pychotherapy, Sweden, June

Balint, M. Ornstein, H. Balint, E. (1985). Psicoterapia Focal, Barcelona: Editorial Gedisa. 5.

Braier, E. (1981). Psicoterapia de Orientación Psicoanalítica. Buenos Aires: Ediciones Nueva Visión.

Etchegoyen, H (1986); los fundamentos de la técnica psicoanalítica; Amorrortu.

Fiorini, H. (1978). Teoría y Técnicas de Psicoterapia, Buenos Aires: Ediciones Nueva Visión.

Freud, S. (1911-1913). Sobre la dinámica de la transferencia (1912). Obras Completas, Vol. XII. Buenos Aires, Amorrortu Editores, 1980.

Freud, S. (1914/2012). Observaciones sobre el amor de transferencia. En: S. Freud: Obras completas. Editorial Biblioteca Nueva.

Gaceta Médica (2020). Salud Mental ¿La próxima pandemia?, pag, https://gacetamedica.com/inv 
estigacion/salud-mental-laproxima-pandemia/.

Green, A. (2003). Ideas directrices para el psicoanálisis contemporáneo. Amorrortu.

Jaime Bacile, Eliana y Cura, Virginia Liliana (2015). El cuerpo del analista. Presencia encuerpo. VII Congreso Internacional de Investigación y Práctica Profesional en Psicología XXII Jornadas de Investigación XI Encuentro de Investigadores en Psicología del MERCOSUR. Facultad de Psicología - Universidad de Buenos Aires, Buenos Aires.

Kernberg, O. (1998). Psicoanálisis, Psicoterapia Psicoanalítica y Psicoterapia de Apoyo: Controversias

Contemporáneas. Revista de Psiquiatría de Uruguay, Vol. 64, No. 2.

Lacan, J. (1975 a) Función y Campo de la Palabra y el Lenguaje en Psicoanálisis. En Escritos 1. México. Siglo Veintiuno.

Lacan, J. (1981): Seminario VIII. La transferencia en su disparidad subjetiva. Versión Anónima.

Laplanche J. y Pontalis J. (1967) Diccionario de psicoanálisis. Buenos Aires. Paidós

Lopera, J., Ramírez, C., Zuluaga, M., \& Ortiz, J. (2010). El método analítico. Medellín: Centro de Investigaciones Sociales y
Humanas (CISH) de la Universidad de Antioquia.

Novoa Cota V., (1999). Esbozo de la Conceptualización Lacaniana sobre la presencia del psicoanalista, Revista Electronica de Psicologia Iztacala. Universidad Nacional Autónoma de México.

Ons S, Delgado O \& Lombardi G, Debate sobre las terapias vía internet, el análisis en tiempos de cuarentena., entrevistados por Oscar Ranzani. Página 12 / 26 de marzo de (2020)

Ramírez, C. (2011). "Orientación psicoanalítica en la psicología". Artículo 19. Apuntes. Grupo de investigación El método analítico y sus aplicaciones en las ciencias sociales $y$ humanas.

Ramírez, Castro, Lerma, Yela, Escobar; (2020). Consecuencias de la Pandemia Covid-2019 en la Salud mental por el aislamiento social. https://preprints.scielo.org/ind ex.php/scielo/preprint/view/30 3

Ruíz, J. J. (2013). Transferencia y contratransferencia. Del Psicoanálisis a la Psicoterapia Analítica Funcional. REALITAS, Revista de Ciencias Sociales, Humanas y Artes, 1 (2), 52-58 
Unzueta Nostas, Carla, \& Lora, María Elena. (2003). EL estatuto del cuerpo en psicoanálisis. Ajayu Órgano de Difusión Científica del Departamento de Psicología UCBSP, 1(1), 136-154. Recuperado en 01 de mayo de 2021, de http://www.scielo.org.bo/sciel o.php?script=sci_arttext\&pid= S207721612003000100009\&lng=es \&tlng=es.

Vaimberg, R. (2010). Psicoterapias tecnológicamente mediadas (PMT). Estudio de procesos y microprocesos a partir de participación online (Tesis doctoral). Archivos TDX. http://www. tesisenxarxa. net/TDX-1019110- 140640

Winnicott D (1994) Juego y realidad. Barcelona: Gedisa, pág. 32. 